view how the blood circulation inside and outside of bones are interconnected to mediate functional or dysfunctional immunity.

Disclosure of Interests: None declared

DOI: 10.1136/annrheumdis-2019-eular.8490

\section{SP0107 THE 4-D+ NANOSCOPE: THE RESOLUTION FOR BIOLOGICAL SAMPLES}

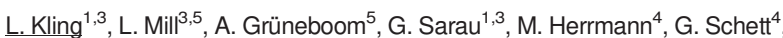
A. Maier ${ }^{5}$, S.H. Christiansen ${ }^{1,2,3}$. ${ }^{1}$ Helmholtz-Zentrum Berlin für Materialien und Energie, Forschergruppe Christiansen, Berlin, Germany, ${ }^{1}$ Christiansen Research Group, Helmholtz Zentrum Berlin für Materialien und Energie, Hahn-Meitner-Platz 1, 14109 Berlin, Germany; ${ }^{2}$ Physics Department, Free University, Berlin, Arnimallee 14, 14195 Berlin, Germany; ${ }^{3}$ Max Planck Institute for the Science of Light, Günther-Scharowsky-Str. 1, 91058 Erlangen, Germany;

${ }^{4}$ Universitätsklinikum der Friedrich-Alexander-Universität Erlangen-Nürnberg, Med 3, Ulmenweg 18, 91054 Erlangen, Germany; ${ }^{5}$ Friedrich-Alexander-Universität Erlangen-Nürnberg, Pattern Recognition Lab, Martensstr. 3, 91058 Erlangen, Germany

Background: In ageing societies all over the globe, the number of people suffering from bone disease, e.g. osteoporosis (OP) in the first place, has increased dramatically. OP considerably impairs patients' life quality, and results in high societal costs. However, current understanding of OP is still insufficient due to the lack of appropriate high resolution tools that permit a thorough analysis of scale bridging bone architectures from macro to nano with statistical significance to support the development of better treatments by drugs or surgical intervention.

Objectives: To revolutionize our knowledge of bone diseases based on an increase in understanding of the underlying bone anatomy, cutting-edge correlative high-resolution microscopy and spectroscopy together with advanced data analysis including machine learning approaches permit reaching the next, so far unprecedented level of understanding.

Methods: Correlative workflows starting from X-ray microscopy (XRM) volume analysis, with voxel sizes of $<1 \mu \mathrm{m}$, over light-sheet fluorescence microscopy and large scale scanning electron microscopy data acquisition, to dual beam microscope analysis (focused electron- and ion beams) permit the scale bridging investigation of bone architectures and thus merging the "big picture" and the underlying ultrastructure with statistical significance. In combination with additional analytical add-ons to these microscopes, physical properties such as optical, mechanical, compositional, structural etc. deliver a highly detailed correlative dataset of bone (cf. Figure 2).

The present paper demonstrates novel findings related to various partly age related bone diseases using advanced correlative data acquisition (cf. Figure 2).

Results: Figure 1 shows the different bone fine structures, composed of trabecular and vascular networks as well as a three-dimensional arrangement of osteolacunae that host osteocytes, as obtained by volume analysis in a new generation of lab-based x-ray microscope (Zeiss XRM Versa 520). These fine structures can be assessed quantitatively with statistical significance and can be correlated with additional modalities as shown in Figure 2. Elucidating examples demonstrating the power of such an approach will be given [ref 1, ref 2, ref 3, ref 4].

Figure 2 shows the an example correlative workflow from sample collection over sample preparation and the acquisition of various image modalities utilizing correlated data from electron-, ion-beam imaging and analytics, probes and focused laser light to study scale bridging bone architectures. We will demonstrate how these correlative workflows will permit to advance the current understanding of bone architectures and function substantially and will show some first systematic correlative microscopy and spectroscopy studies on mouse tibia and human bone [ref1, ref2, ref3, ref 4].

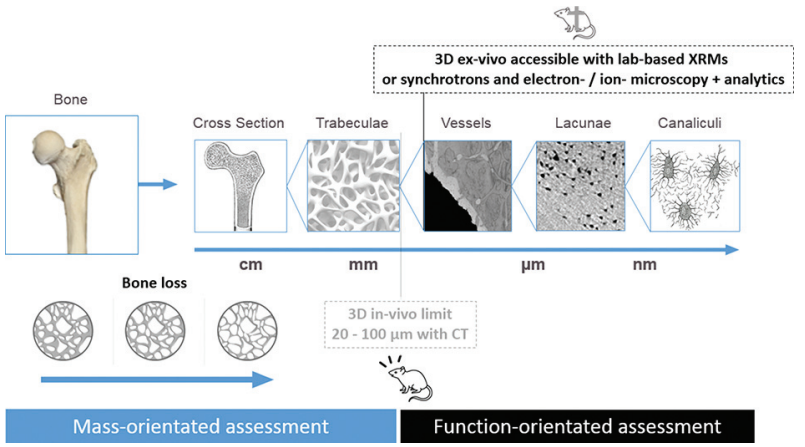

Abstract SP0107 Figure 1. Bone anatomy with scale - bridging architecture: mass oriented assessment with conventional methods such as DEXA and $\mu \mathrm{CT}$ and function-oriented assessment using correlative microscopy including volume analysis of trans-cortical vessels, osteo-lacunae and canaliculi by XRM and FIB/SEM.
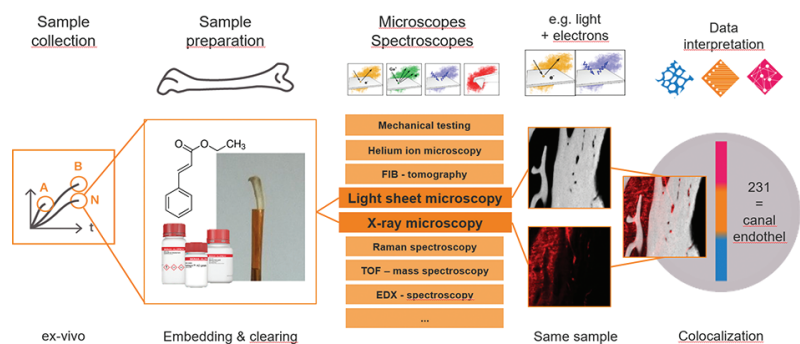

Abstract SP0107 Figure 2. Correlative microscopy/spectroscopy workflow in bone research including (i) the challenges of sample preparation, (ii) the interplay of various image and data modalities, (iii) data evaluation, resolution enhancement and data analysis based on deep learning strategies; finally, new insights in open questions related to bone remodelling and bone structure at various length scales in relation to vasculature will be extracted from statistical correlative microscopy data.

Conclusion: Based on our correlative analytics and the data availability, the advanced data interpretation using machine learning approaches will be dis cussed. Using this approach, all details of bone micro-/nano-architecture can be used to provide a novel clinical tool-set for future for early detection of bone diseases.

\section{REFERENCES}

[1] P. Milovanovic, et al., Bone tissue aging affects mineralization of cement lines, bone 110, 187 (2018).

[2] A. Grüneboom, et al., A network of trans-cortical capillaries forms the mainstay for blood circulation in long bones, Nature Metab. Published online (02/2019).

[3] P. Milovanovic, et al., The Formation of Calcified Nanospherites during Micropetrosis Represents a Unique Mineralization Mechanism in Aged Human Bone, small 13(3), 1602215 (2017).

Disclosure of Interests: None declared

DOI: 10.1136/annrheumdis-2019-eular.8516

\section{SP0108 LSFM AND CATCHING UP IN JOINT IMAGING}

\section{Anika Grueneboom ${ }^{1}$.}

Background: Bone tissue differs from other organs by its hardness and high optical density. These physical characteristics are challenging in the context of fluorescence based imaging studies addressing bone anatomy and physiology. The development of novel imaging technologies, such as light-sheet fluorescence microscopy and X-ray microscopy, and tissue-specific preparation procedures like optical clearing allowed us to reveal a so far overseen network of blood ves sels in the cortical bone. These trans-cortical vessels (TCVs) directly connect the bone marrow vascularization with the periosteum and make the major contribution to total blood flow in long bones. Furthermore, we could show that immune cells are recruited to the peripheral circulation via TCVs and that TCVs are highly remodeled und inflammatory conditions. In case of chronic arthritis we observed a massive increase in TCV numbers, especially at the metaphysis of long bones This increase in bone vascularization confirms observations of previous studies describing increasing numbers of microcanals in the bare area of metacarpal heads in the hands of rheumatoid arthritis patients. These alterations in bone anatomy might hint to an alternative mechanism by which immune cells are recruited into inflamed joint tissue. While current concepts describe the recruitment of immune cells from the systemic circulation, immune cells can potentially infiltrate the arthritic joints directly from the underlying bone marrow.

Objectives: Clarifying the anatomical routes of cell recruitment as well as exploring the regulatory mechanism guiding immune cells from the bone marrow to the site of inflammation might bear novel therapeutic strategies to resolve inflammation and tissue injury in rheumatoid arthritis.

Methods: 3D optical imaging approaches like Light-sheet fluorescence microscopy and 2-Photon laser-scanning microscopy enable the visualization of entire bones and joints. In combination with tissue-specific preparation procedures and labeling strategies inflammatory mediated changes in vascularization and cell recruitment can be analyzed.

Results: The joint tissue architecture is massively affected by inflammatory arthritic processes leading to changes in vascularization, permeability and cellular tissue composition. These processes are directly connected to the onset as well as the resolution of inflammatory arthritis.

Conclusion: Newly available imaging techniques as light-sheet fluorescence microscopy facilitate new insights into bone and joint tissue architecture, function and inflammatory processes. These findings might guide the identification of novel 
proresolving mediators to develop therapeutic strategies for managing tissuedamage-induced inflammation.

\section{REFERENCE:}

[1] Grüneboom, A. et al., A network of trans-cortical capillaries as mainstay for blood circulation in long bones. Nature Metabolism 1, 236-250, doi:10.1038/s42255-018-0016-5 (2019)

Disclosure of Interests: None declared

DOI: 10.1136/annrheumdis-2019-eular.8487

FRIDAY, 14 JUNE 2019

10:15:00 - 11:45:00

\section{Paradigm shifts in arthritides}

\section{SP0109 HOT: DIAGNOSIS AND TREATMENT OF INFECTION RELATED ARTHRITIDES}

Robert Schoen. Yale School of Medicine, Rheumatology, Allergy, Clinical Immunology, New Haven, United States of America

Background: Management of infectious arthritis has evolved because of empiric knowledge, clinical studies, and emerging pathogens. For patients with bacterial arthritis, organism specific, effective anti-microbial therapy is essential, but assessment of co-morbidities, adequate joint drainage, and supportive care are also required. For patients with infectious arthritis an acute intervention is usually necessary, since delay in diagnosis often leads to unsatisfactory outcome. In some situations, the distinction between infection and a post-infectious inflammatory process may be challenging.

Objectives: The goal of this lecture is to review management of septic arthritis in native joints. In addition, I will discuss Lyme arthritis and chronic chikungunya arthritis as examples of the broadening spectrum of infectious arthritis.

Methods: This lecture will rely on my clinical experience and a Pubmed literature search.

Results: In this HOT lecture, I will provide practical, up to date information about the diagnosis and management of septic (bacterial) arthritis of native joints. I will discuss longstanding treatment paradigms, as well as advances in management and areas of uncertainty. I will then consider two emerging infections, Lyme arthritis and chronic chikungunya arthritis, that illustrate the changing spectrum of infectious arthritis.

Conclusion: It is the intention of this lecture to assist rheumatologists in management of patients across the spectrum of infectious arthritis. Accurate diagnosis and treatment are often challenging, but critical to satisfactory patient outcome.

\section{REFERENCES:}

[1] Ross JJ: Septic arthritis of native joints. Infect Dis Clin North Am 2017; 31: 203-218.

[2] Schoen RT: Musculoskeletal manifestations of Lyme disease. In: Basow DS (ed): UptoDate 2019. Waltham, MA.

[3] Kennedy Amaral Pereira J, Schoen RT: Management of chikungunya arthritis. Clin Rheumatol 2017; 36: 2179-2186.

Disclosure of Interests: None declared

DOI: 10.1136/annrheumdis-2019-eular.8583

FRIDAY, 14 JUNE 2019

13:30:00 - 15:00:00

\section{Safety First! Infectious complications and pregnancy issues in patients with rheumatic diseases}

\section{SP0110 \\ WIN: PREGNANCY ISSUES IN PATIENTS WITH RHEUMATIC DISEASES: THE OB PERSPECTIVE FOR RHEUMATOLOGISTS}

Catherine Nelson-Piercy. Guy's and St. Thomas' Foundation Trust and Queen Charlotte's and Chelsea Hospital London, United Kingdom

Pregnancy issues in patients with rheumatic diseases: the OB perspective for rheumatologists

Learning Objectives:
- Understand the importance of pre pregnancy counselling for women with rheumatic disease in pregnancy

- Understand the risk factors for adverse pregnancy outcome in women with rheumatic disease

- Understand the medications which are compatible with use in pregnancy and lactation

- Understand the management of rheumatic disease in pregnancy

Abstract: Rheumatic disease predominantly affects women of child-bearing age and are commonly encountered in obstetric practice. Pregnancy poses an important challenge for doctors looking after these women. Knowledge about medication safety, the effect of pregnancy on the disease, and vice versa, together with pre-conception counselling and multidisciplinary team care, are important to provide the best obstetric and medical care to these women.

Women with rheumatic have increased risks of miscarriage, preterm delivery, preeclampsia, fetal growth restriction, and disease flare in pregnancy. The main risk factor for adverse pregnancy outcomes in inflammatory arthritides is active disease/flare. For women with SLE the risks are lupus nephritis, particularly with CKD class 3-5, anti Ro/La antibodies, active disease and antiphospholipid antibodies. The most important issues of delaying pregnancy until there is quiescent disease, ensuring continued remission by continuation of drugs that are safe in pregnancy and adequately and promptly treating any flare of disease will be dis cussed. Adequate surveillance of the mother and fetus is imperative, but stratification of women is important to ensure that those with low risk pregnancies are not over medicalized.

There is an understandable reluctance to prescribe drugs, particularly immuno suppressant drugs, in pregnancy and in breast feeding mothers. However much harm can result if drugs are withdrawn, omitted or the dose reduced inappropriately. Active disease has an adverse effect on female fertility and time to pregnancy aw well as impacting adversely on pregnancy outcomes.

Guidelines from the British Society of Rheumatology and EULAR have reviewed the safety data for antirheumatic drugs in pregnancy. These publications include recommendations for which drugs are compatible with pregnancy and during lactation. These guidelines should reduce errors of omission where important medication for disease control are discontinued prior to or in pregnancy and empower rheumatologists to help women to time their pregnancies during disease remission and with continuation of medications including biologics compatible with pregnancy.

\section{REFERENCES:}

[1] Ateka-Barrutia O, Nelson-Piercy C. Connective tissue disease in pregnancy. Clin Med. 2013; 13: 580-4. Soh MC, Nelson-Piercy C. High-risk pregnancy and the rheumatologist. Rheumatology (Oxford). 2015 Apr;54(4):572-87. doi: 10.1093/rheumatology/keu394. Epub 2014 Dec 3.

[2] Flint J, Panchal S, Hurrell A, van de Venne M, Gayed M, Schreiber K, Arthanari S, Cunningham J, Flanders L, Moore L, Crossley A, Purushotham N, Desai A, Piper M, Nisar M, Khamashta M, Williams D, Gordon C Giles I; BSR and BHPR Standards, Guidelines and Audit Working Group. $\mathrm{BSR}$ and BHPR guideline on prescribing drugs in pregnancy and breastfeeding-Part I: standard and biologic disease modifying anti-rheumatic drugs and corticosteroids. Part II: analgesics and other drugs used in rheumatology practice. Rheumatology (Oxford). 2016 Jan 10. pii: kev405.

[3] Götestam Skorpen C, Hoeltzenbein M, Tincani A, Fischer-Betz R, Elefant E, Chambers C, da Silva J, Nelson-Piercy C, Cetin I, Costedoat-Chalu meau N, Dolhain R, Förger F, Khamashta M, Ruiz-Irastorza G, Zink A Vencovsky J, Cutolo M, Caeyers N, Zumbühl C, Østensen M. The EULAR points to consider for use of antirheumatic drugs before pregnancy, and during pregnancy and lactation. Ann Rheum Dis. 2016 May;75(5):795-810. doi: 10.1136/annrheumdis-2015-208840. Epub 2016 Feb 17.

Disclosure of Interests: Catherine Nelson-Piercy Consultant for: UCB, Speak ers bureau: UCB

DOI: 10.1136/annrheumdis-2019-eular 8549

FRIDAY, 14 JUNE 2019

13:30:00 - 15:00:00

\section{Immunosuppression in SSc - a matter of timing!}

\section{SP0111 POTENTIAL CELLULAR AND MOLECULAR TARGETS}

Jörg Distler. University of Erlangen-Nuremberg, Department of Internal Medicine 3 , Erlangen, Germany

The pathophysiology of systemic sclerosis (SSc) is characterized by a cascade of microvascular injury with apoptosis of endothelial cells, Th2/M2 biased 